\title{
Lateral gene transfer between obligate intracellular bacteria: Evidence from the Rickettsia massiliae genome
}

\author{
Guillaume Blanc, ${ }^{1,3}$ Hiroyuki Ogata, ${ }^{1}$ Catherine Robert, ${ }^{2}$ Stéphane Audic, ${ }^{1}$ \\ Jean-Michel Claverie, ${ }^{1}$ and Didier Raoult ${ }^{2}$ \\ ${ }^{1}$ Structural and Genomic Information Laboratory, CNRS-UPR 2589, Institut de Biologie Structurale et Microbiologie, IFR 88, Parc \\ Scientifique de Luminy, 13288 Marseille Cedex 9, France; ${ }^{2}$ Unité des Rickettsies, CNRS-UMR 6020, IFR 48, Faculté de Médecine, \\ 13385 Marseille Cedex 5, France
}

\begin{abstract}
Rickettsia massiliae is a tick-borne obligate intracellular $\alpha$-proteobacteria causing spotted fever in humans. Here, we present the sequence of its genome, comprising a 1.3-Mb circular chromosome and a 15.3-kb plasmid. The chromosome exhibits long-range colinearity with the other Spotted Fever Group Rickettsia genomes, except for a large fragment specific to $R$. massiliae that contains 14 tra genes presumably involved in pilus formation and conjugal DNA transfer. We demonstrate that the tra region was acquired recently by lateral gene transfer (LGT) from a species related to Rickettsia bellii. Further analysis of the genomic sequences identifies additional candidates of LGT between Rickettsia. Our study indicates that recent LGT between obligate intracellular Rickettsia is more common than previously thought.
\end{abstract}

[Supplemental material is available online at www.genome.org.]

The Rickettsia genus, which includes Rickettsia massiliae, is a group of arthropod-borne, obligate intracellular alphaproteobacteria (Raoult and Roux 1997). This genus has been classified into three groups, namely, the typhus group (TG), which includes Rickettsia prowazekii and Rickettsia typhi, the spotted fever group (SFG), which includes Rickettsia rickettsii and Rickettsia conorii, and the Rickettsia bellii group (Stothard et al. 1994; Roux et al. 1997; Blanc et al. 2007). The SFG rickettsiae are further divided in two subgroups: the Rickettsia conorii subgroup and the $R$. massiliae subgroup, the latter also including Rickettsia montanensis, Rickettsia aeschlimannii, and Rickettsia rhipicephali (Roux et al. 1997; Fournier et al. 1998). Natural resistance to rifampin is a phenotypic marker of the $R$. massiliae subgroup (Drancourt and Raoult 1999), whereas rickettsiae belonging to the $R$. conorii subgroup and the TG rickettsiae are naturally susceptible to rifampin (Rolain et al. 1998).

Many SFG rickettsiae infect and multiply in almost all organs of ticks, in particular the salivary glands, which enable the bacteria to be transmitted to vertebrate hosts during feeding (Raoult and Roux 1997). Rickettsia massiliae was first isolated from Rhipicephalus sanguineus collected in Marseille (France) in 1992 (Beati and Raoult 1993). Since then, this rickettsiae has been commonly detected in Rhipicephalus ticks from Central Africa, France, Greece, Mali, Portugal, Spain, Switzerland, and the United States (Matsumoto et al. 2005; Eremeeva et al. 2006). It is also transmitted transovarially, at least in Rhipicephalus turanicus (Matsumoto et al. 2005). R. massiliae might be commonly associated with these worldwide-distributed ticks. $R$. massiliae has also been recently identified in Ixodes ricinus ticks (FernandezSoto et al. 2006). At the beginning of the 20th century, SFG rickettsiae were recognized as human pathogens. Although SFG rickettsioses are among the oldest known vector-borne diseases, they are also now recognized as emerging infections of humans

${ }^{3}$ Corresponding author.

E-mail guillaume.blanc@igs.cnrs-mrs.fr; fax 33-4-91-82-54-21. Article published online before print. Article and publication date are at http:// www.genome.org/cgi/doi/10.1101/gr.6742107.
(Parola et al. 2005). In Spain where R. massiliae is prevalent in ticks, serological studies (Cardenosa et al. 2003) and resistance to rifampin make this bacterium a putative agent of spotted fever. Recently, R. massiliae has been reported as a human pathogen (Vitale et al. 2005).

The rickettsiae have comparatively small genomes (1.1-1.5 $\mathrm{Mb}$ ) that have arisen through a recent and ongoing genome degradation process (Andersson et al. 1998). To date, 10 complete Rickettsia genome sequences have been deposited in public databases. They include the genomes of two R. bellii strains (GenBank AARC00000000; Ogata et al. 2006), two sequences from the TG rickettsiae ( $R$. prowazekii [Andersson et al. 1998] and $R$. typhi [McLeod et al. 2004]), five sequences from the SFG group $(R$. conorii [Ogata et al. 2001], Rickettsia sibirica [Malek et al. 2004], $R$. rickettsii [GenBank AADJ00000000], Rickettsia felis [Ogata et al. 2005] and Rickettsia akarii [GenBank AAFE00000000]), and the sequence of Rickettsia canadensis (Eremeeva et al. 2005) for which the phylogenetic position is unclear. Here, we report the sequencing and the primary analysis of the genome of $R$. massiliae strain MTU5 isolated from the R. turanicus tick collected on horses in Camargues, France. Recently, our group used seven Rickettsia species (including R. massiliae) in a large-scale comparative genomic study to investigate the evolutionary processes that shaped the chromosomes of the genus (Blanc et al. 2007). In particular, our results suggested a lack of recent horizontal gene transfer between Rickettsia species and other bacteria. In the present analysis, a different approach allowed us to pinpoint horizontal gene transfers between Rickettsia species that were not discovered previously.

\section{Results}

Highlights of the $R$. massiliae genome

The genome of $R$. massiliae is made of two replicons: a 1,360,898bp circular chromosome, and a 15,286-bp circular plasmid. After 
$R$. felis (Ogata et al. 2005), R. massiliae is the second sequenced Rickettsiales harboring a plasmid. We detected 968 protein genes on the chromosome as well as 212 pseudogenes. Only 26 gene products, including 21 transposases, exhibited no reciprocal best-BLAST hit in the other Rickettsia species (BLASTP Evalue $<1 \times 10^{-5}$ ). The plasmid is predicted to contain 12 protein genes and a pseudogene, among which seven have homologs on the $R$. felis plasmid and two others are related to transposable elements. Thus, the $R$. massiliae genome appears to encode only a limited number of novel functions with respect to the other Rickettsia. A more detailed description of the genomic features of $R$. massiliae is presented in the Supplemental Material.

As expected from their close relatedness (identity $>98 \%$ in coding sequence), the $R$. massiliae and $R$. conorii chromosomes exhibit a high level of colinearity (Fig. 1). However, a particular $54.6-\mathrm{kb}$ segment retained our attention because it is absent in $R$. conorii. This region contains 44 genes, including 14 tra-related genes that probably encode components of a type IV secretion system (T4SS) for conjugal DNA transfer (Ogata et al. 2005, 2006). The relatively high number of specific transposase genes and pseudogenes (36 sequences) suggests that $R$. massiliae underwent a recent expansion of transposons, a phenomenon also encountered in the chromosomes of $R$. bellii (39 sequences) and $R$. felis (66 sequences) but not in the other sequenced Rickettsia genomes. Interestingly, the R. massiliae, $R$. bellii, and $R$. felis genomes contain several tra genes. The recently released genome of Orientia tsutsugamushi (Cho et al. 2007), a remote relative of Rickettsia species, exhibits also very high numbers of transposase ( $>400$ genes) and tra genes (359 genes). Thus, there may be a link between the relative abundance of transposase genes in the Rickettsiale genomes and an active process of conjugation enabling the acquisition of foreign DNA.

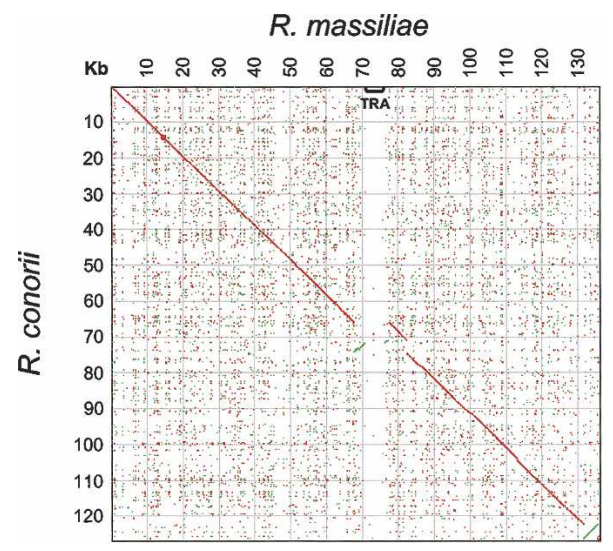

Figure 1. Dot-plot comparison of the R. massiliae and R. conorii genomes. Red and green dots represent forward and reverse BLASTN matches, respectively $\left(E\right.$-value value $<1 \times 10^{-5}$ ). Position of the tra cluster is indicated in gray.

\section{The tra gene cluster}

The $R$. massiliae tra region is most similar to that of $R$. bellii in terms of gene order and content (Fig. 2). The two segments share 25 ORFs, including the entire set of 14 tra-related genes and 11 ORFs encoding five unknown proteins; a site-specific DNA adenine methylase, dam-like; an integrase; a signal transduction histidine, kinase-like; and a spoT family protein (Table 1). In addition, both species contain specific genes: the $R$. massiliae tra cluster contains 21 extra genes (genes for 13 transposases, four unknown proteins, a toxin of a toxin-antitoxin system, a phageassociated protein, a protein similar to the DNA polymerase III alpha chain, and an archaeal ATPase family protein), and R. bellii contains three specific genes (genes for a leucine-rich protein, a cassette chromosome recombinase B, and spoT16). The tra genes are absent in the other sequenced Rickettsia chromosomes except for $R$. canadensis and Rickettsia africae, which contain small subsets of the $R$. massiliae tra cluster genes (Fig. 2; Table 1). The genes surrounding the tra region are conserved in sequence and order in most Rickettsia species, even in those that do not possess a tra cluster region (Supplemental Figs. S1-S7).

In both $R$. massiliae and $R$. bellii, the tra cluster is flanked by an integrase gene (tyrosine recombinase family) and a $R$ RNA-Val gene. Many integrases specify integration of prokaryotic genetic elements into tRNA or tmRNA genes (Williams 2002). To avoid deactivation of the recipient gene (e.g., tRNA), the foreign genetic element usually carries at its integration site the same segment of gene as the one displaced. After integration, the host tRNA is then split into two pieces lying at each extremity of the integrated genetic element (see Fig. 1 in Williams 2002 for details). Consistent with such an integration process, we identified a 51-bp sequence highly similar to the 3 '-extremity of the $t R N A$ $\mathrm{Val}$, located $90 \mathrm{bp}$ upstream from the $R$. massiliae integrase gene (coordinates 707,321-707,371). In $R$. bellii, the 40-bp long displaced $t R N A$ - $\mathrm{Val}$ segment lies $395 \mathrm{bp}$ upstream from the integrase gene (coordinates: 498,401-498,440). Thus, the tra regions in $R$.

\section{Genome Research \\ www.genome.org}


Table 1. Genes of the R. massiliae tra cluster region and their homologs in other Rickettsia

\begin{tabular}{|c|c|c|c|c|c|c|c|c|}
\hline R. massiliae ${ }^{a}$ & R. bellii & R. africa $e^{\mathrm{a}, \mathrm{b}}$ & R. canadensis $^{\mathrm{a}}$ & $\begin{array}{l}\text { R. felis } \\
\text { plasmid }^{\text {a }}\end{array}$ & R. felis ${ }^{\mathrm{a}}$ & R. rickettsii & R. akarii & Predicted function \\
\hline RMA_0713 & RBE_0440 & & & & & & & Integrase \\
\hline RMA_0714 & & & & & & & & Phage-associated protein \\
\hline RMA_0715 & & & & & & & & Toxin of TA system \\
\hline & RBE_0439 & & & & & & & Leucine-rich repeat protein \\
\hline RMA_0716 & RBE_0438 & & & & & & & Unknown \\
\hline RMA_0717 & RBE_0437 & & & & & & & Conjugal transfer protein $\mathrm{TraE}_{\mathrm{F}}$ \\
\hline RMA_0718 & RBE_0436 & & & & & & & Unknown \\
\hline RMA_0719 & RBE_0435 & & & & & & & Unknown \\
\hline RMA_0720 & RBE_0434 & & RcanM_01000520 & & & & & $\mathrm{F}$ pilus assembly protein $\mathrm{TraB}_{\mathrm{F}}$ \\
\hline RMA_0721 & RBE_0433 & & & & & & & Conjugal transfer protein $\mathrm{TraV}_{\mathrm{F}}$ \\
\hline RMA_0722 & RBE_0432 & & & & & & & Conjugal transfer protein $\mathrm{TraC}_{\mathrm{F}}$ \\
\hline RMA_0723 & & & & & & & & Archaeal ATPase family protein \\
\hline & & & RcanM_01000516 & & & & & Unknown \\
\hline $\begin{array}{l}\text { RMA_0724 } \\
\text { RMA_0725 }\end{array}$ & RBE_0431 & & & & & & & Conjugal transfer protein TraW $_{F}$ \\
\hline RMA_0726 & RBE_0430 & & & & & & & Conjugal transfer protein $\mathrm{TraU}_{\mathrm{F}}$ \\
\hline RMA_0727 & RBE_0429 & & & & & & & Conjugal transfer protein $\mathrm{TrbC}_{\mathrm{F}}$ \\
\hline RMA_0728 & RBE_0428 & & & & & & & Conjugal transfer protein $\mathrm{TraN}_{\mathrm{F}}$ \\
\hline RMA_0729 & RBE_0427 & & & & & & & $\mathrm{F}$ pilus assembly protein $\mathrm{TraF}_{\mathrm{F}}$ \\
\hline RMA_0730 & RBE_0426 & & & & & & & $\mathrm{F}$ pilus assembly protein $\mathrm{TraH}_{\mathrm{F}}$ \\
\hline RMA_0731 & RBE_0425 & & RcanM_01000514 & pRF46 pRF47 & & & & Conjugal transfer protein $\mathrm{TraG}_{\mathrm{F}}$ \\
\hline RMA_0732 & RBE_0424 & & $\begin{array}{l}\text { RcanM_01000512 } \\
\text { RcanM_01000513 }\end{array}$ & pRF26 pRF17 & & & & $\begin{array}{l}\text { Tetratricopeptide } \\
\text { repeat-containing protein }\end{array}$ \\
\hline RMA_0733 & RBE_0423 & raf_ORF0650 & $\begin{array}{l}\text { RcanM_01000511 } \\
\text { RcanM_01000519 }\end{array}$ & pRF43 pRF44 & & & & Conjugal transfer protein $\operatorname{TraD}_{\mathrm{F}}$ \\
\hline RMA_0734 & RBE_0422 & raf_ORF0651 & & pRF41 & & & & Transposase \\
\hline RMA_0735 & RBE_0421 & & $\begin{array}{l}\text { RcanM_01000506 } \\
\text { RcanM_01000507 } \\
\text { RcanM_01000509 } \\
\text { RcanM_01000510 }\end{array}$ & pRF38 pRF39 & & & & Conjugal transfer protein $\mathrm{TraA}_{\mathrm{Ti}}$ \\
\hline RMA_0736 & & & & & & & & Transposase \\
\hline RMA_0737 & & & & & & & & Transposase \\
\hline RMA_0738 & & & & & & & & Transposase \\
\hline RMA_0739 & & & & & & & & Unknown \\
\hline RMA_0740 & & & & & & & & Transposase \\
\hline RMA_0741 & & & & & & & & Transposase \\
\hline RMA_0742 & & & & & & & & Transposase \\
\hline RMA_0743 & & & RcanM_01000500 & & & & & Transposase \\
\hline RMA_0744 & RBE_0418 & & & & & & & Unknown \\
\hline RMA_0745 & & & & & & & & Transposase \\
\hline RMA_0746 & & & & & & & & Transposase \\
\hline RMA_0747 & & & & & & & & Transposase \\
\hline RMA_0748 & & & RcanM_01000500 & pRF49 & & & & Transposase \\
\hline RMA_0749 & & & & & & & & Transposase \\
\hline RMA_0750 & & & & & & & & Transposase \\
\hline RMA_0751 & & & & & & & & DNA polymerase III alpha chain \\
\hline \multirow[t]{6}{*}{ RMA_0752 } & RBE_0420 & & RcanM_01000504 & pRF37 & & & & Conjugal transfer protein $\mathrm{TraD}_{\mathrm{Ti}}$ \\
\hline & & & $\begin{array}{l}\text { RcanM_01000503 } \\
\text { RcanM_01000502 }\end{array}$ & & $\begin{array}{l}\text { RF_0385 } \\
\text { RF_0386 }\end{array}$ & & & $\begin{array}{l}\text { Proline/betaine transporter } \\
\text { Unknown }\end{array}$ \\
\hline & & raf ORF0652 & RcanM 01000501 & & RF 0384 & & & ppGpp hydrolase spoT15 \\
\hline & & & & & RF_0383 & & & Transposase \\
\hline & & & & & RF_0382 & & & Transposase \\
\hline & RBE_0419 & $\begin{array}{l}\text { raf_ORF0653 } \\
\text { raf_ORF0654 }\end{array}$ & & & RF_0381 & & & ppGpp synthetase spoT16 \\
\hline RMA_0753 & RBE_0417 & $\begin{array}{l}\text { raf_ORF0655 } \\
\text { raf_ORF0656 }\end{array}$ & & & RF_0380 & & & ppGpp hydrolase spoT6 \\
\hline RMA_0754 & RBE_0416 & $\begin{array}{l}\text { raf_ORF0657 } \\
\text { raf_ORF0658 }\end{array}$ & & & & & & $\begin{array}{l}\text { Signal transduction } \\
\text { histidine kinase }\end{array}$ \\
\hline RMA_0755 & RBE_0415 & raf_ORF0659 & & & & & & \\
\hline RMA_0756 & RBE_0414 & raf_ORF0660 & $\begin{array}{l}\text { RcanM_01000498 } \\
\text { RcanM_01000499 }\end{array}$ & pRF19 & & Rick02000935 & Pseudogene & Unknown (U gene) \\
\hline tRNAVal & tRNAVal & tRNAVal & tRNAVal & & & tRNAVal & tRNAVal & Anticodon: GAC \\
\hline
\end{tabular}

aGenes comprising several ORFs in a species correspond to split pseudogenes.

bThe $R$. africae gene annotation is from Blanc et al. (2007) and is available at http://www.igs.cnrs-mrs.fr/mgdb/Rickettsia/rig/. 
massiliae and $R$. bellii probably result from the integration of a site-specific integrative and conjugative element (Burrus et al. 2002). The organization of the rickettsial tra regions is very similar to that of the tra clusters of O. tsutsugamushi (Cho et al. 2007), including tra-associated genes such as transposase, spoT, Histidine Kinase, and Dam. Overall, 29 of the 44 R. massiliae tra cluster genes have homologs in the O. tsutsugamushi genome. This points to a probable common origin of those genetic elements.

The nucleotide sequence immediately downstream of the recombined $R$. massiliae tRNA-Val gene (toward the RMA_0756 gene) is part of the original conjugative element that gave birth to the chromosomal tra cluster. We searched for similar sequences in the downstream region of the $t R N A$-Val gene in all sequenced Rickettsia genomes. Significant matches (BLASTN Evalue $<1 \times 10^{-5}$ ) were identified not only for $R$. bellii, $R$. africae, and $R$. canadensis (as expected) but also for $R$. rickettsii and $R$. akarii, over regions that extend into the first gene (RMA_0756; referred to hereafter as the $U$ gene) of the $R$. massiliae conjugative element (Supplemental Fig. S8). Thus, these two latter species appear to contain traces of ancient integrated genetic elements, at least partly related to that of $R$. massiliae. Out of the Rickettsia genus, homologs of the $U$ gene are only found in the $O$. tsutsugamushi genome (35 copies, BLASTP $E$-value $<10^{-10}$ ). In contrast, homologs are present in the $R$. massiliae (RMA_p01) and $R$. felis (pRF19) plasmids and in the $R$. bellii (RBE_1035) and the $R$. prowazekii (pseudogene between $R P 708$ and RP709) genomes. The $R$. prowazekii $U$ pseudogene is located in a 12 -kb region specific to R. prowazekii (McLeod et al. 2004), which also includes several transposase ( $R P 710$ and $R P 715$ ) and recombinase pseudogenes (RP711) that are uniquely found on both the $R$. felis and $R$. massiliae plasmids (Supplemental Fig. S9). In addition, this region contains a fragment of a patatin-like protein gene mostly similar to the pat2 gene of the $R$. felis plasmid. This region might also result from the integration of a genetic element or plasmid. We also note that homologs of spoT6, spoT16, spoT15, and the unknown protein gene RcanM01000502 (R. canadensis) are organized in cluster in the $R$. felis genome, in the same order as in the consensus structure of the Rickettsia tra regions (Table 1). However, they lie at a location remote from the tRNA-Val gene, suggesting that they do not originate from the same genetic element. Thus, this analysis supports the view that integrated genetic elements are relatively widespread in Rickettsia, at least in the form of traces.

\section{Phylogenetic study of the tra cluster genes}

A previous study of the $R$. bellii genome showed that the closest homologs of the tra genes outside the Rickettsia genus were found in microbes living inside amoebae, including Legionella pneumophila and Protochlamydia amoebophila (Ogata et al. 2006). Here we present the phylogenetic relationships of the tra-cluster genes among the Rickettsia species. Phylogenetic reconstructions were conducted for 13 homologous gene families for which sequences were present in at least three distinct Rickettsia species and with sufficient overlap (protein alignment $>75$ sites). The $R$. felis plasmid proteins were generally difficult to align with their rickettsial homologs, suggesting that the plasmid sequences are the most distantly related. This is confirmed in six phylogenetic reconstructions where plasmid proteins are separated by the longest branches from the rest of the rickettsial sequences (Fig. 3C-H). In all cases but in the transposase tree (Fig. 3F), the plasmid proteins
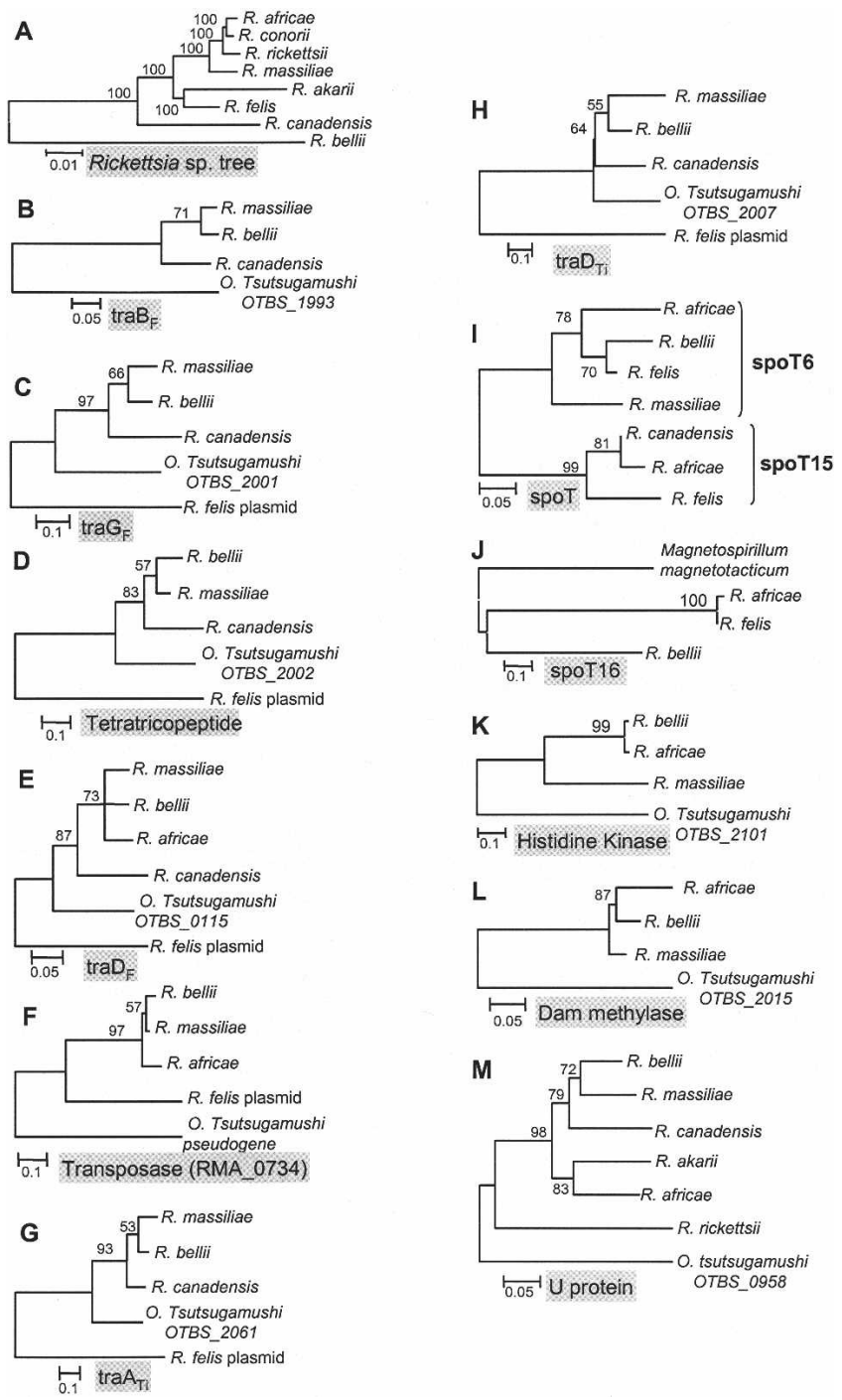

Figure 3. Phylogenetic relationships of the tra cluster genes. $(A-M)$ Phylogenetic trees are constructed from protein sequences using the maximum-likelihood method. Models of substitution that best fit the data are as follows: JTT for $D$ and $M$; JTT $+\Gamma$ for $C, E$, and $J ; J T T+I$ for $K$; WAG $+\Gamma$ for $G$; WAG $+\Gamma+I$ for $A$; CpREV for $B, F$, and $L ; C$ PREV $+\Gamma$ for l; RtREV for $H$. The Rickettsia species tree $(A)$ is constructed from the concatenated alignment of 38 ribosomal proteins (6209 sites). Gene names and branch length scales (number of substitution per site) are shown below trees. Bootstrap support values, calculated over 500 alignment replicates, are given beside branches. Consecutive ORFs of pseudogenes (Table 1 ) were concatenated for the analysis. In addition to the Rickettsia sequences, the O. tsutsugamushi best BLASTP hit was included in the phylogenetic reconstructions when available ( $E$ value $<1 \times 10^{-10}$ )

are more distant from their rickettsial chromosomal counterparts than are the O. tsutsugamushi homologs.

In seven phylogenetic trees, the $R$. massiliae proteins are the closest homologs of the $R$. bellii sequences (Fig. 3C-H,M). This phylogenetic affinity is consistent with the high level of synteny between the two regions but in disagreement with the species phylogeny (Fig. 3A), in which $R$. bellii is the earliest diverging species and $R$. massiliae is anchored within the spotted fever group (together with $R$. africae and $R$. rickettsii). Exceptions to this rule are spoT6 (Fig. 3I), histidine kinase (Fig. 3K), and dam methylase

\section{Genome Research}

www.genome.org 
(Fig. 3L), for which the R. bellii sequences are more closely related to $R$. africae or $R$. felis than $R$. massiliae. This phylogenetic proximity is also in disagreement with the species tree. The $U$ gene exhibits even more phylogenetic incongruence: the SFG rickettsiae ( $R$. africae, $R$. massiliae and $R$. rickettsii) fall in three distinct lineages (Fig. 3M). The position of $R$. canadensis with respect to $R$. massiliae and $R$. bellii is always consistent across the phylogenetic trees of the tra proteins and the $U$ protein, in which it stands out as a sister group (Fig. 3B$\mathrm{E}, \mathrm{G}, \mathrm{H}, \mathrm{M})$.

The striking picture emerging from this analysis is that none of the tra cluster proteins seems to present an evolutionary history compatible with a strict vertical inheritance. Only two protein families (histidine kinase and $U$ gene) formally rejected $(\alpha=5 \%)$ the species tree topology using the ShimodairaHasegawa (SH) test (Shimodaira and Hasegawa 1999) (Supplemental Table S1). The poor discrimination of the SH tests between alternative topologies is likely the consequence of the short length of some alignments and/or the lack of strong phylogenetic signal. The departure from the species phylogeny implies that the tra-regions were subject to horizontal transfer events between rickettsiae, but other less likely scenarios such as a complex interplay of duplication/differential loss can also be invoked. In this respect, the phylogenetic relationships between the $U$ genes (Fig. $3 \mathrm{M}$ ) are particularly interesting because they suggest that the genetic elements (or traces of thereof) result from different integration events, many of which occurred in the same tRNA-Val gene due to the integrase site specificity. Furthermore, the different genes did not produce consistent phylogenies in spite of their collinear organization. However, we note that the phylogenies of the tra genes and the transposase (Fig. 3B-H) that lie in the $5^{\prime}$ part of the tra-cluster are consistent between each other (albeit with some low bootstrap supports), whereas the genes corresponding to the $3^{\prime}$ extremity of the tra-region (spoT, histidine kinase, dam, and U protein; Fig. 3I-M) exhibit heterogeneous evolutionary histories. This suggests that the original genetic elements result from the assembly of gene modules with different evolutionary trajectories, a phenomenon frequently observed in conjugative genetic elements (Burrus et al. 2002).

\section{Horizontal transfer between $R$. massiliae and $R$. bellii}

We determined the level of synonymous substitutions $\left(K_{\mathrm{s}}\right)$ between pairs of putative orthologous genes in $R$. bellii and $R$. massiliae. All but three gene pairs falling in the tra cluster exhibit a smaller level of divergence $\left(K_{\mathrm{s}}\right.$ values from 0.01 to 0.69$)$ than the gene pairs in the rest of the genome (overall median $K_{\mathrm{s}}$ value of 1.58; Fig. 4A). This indicates that those tra cluster genes were not vertically inherited from the $R$. bellii and $R$. massiliae common ancestor but rather diverged more recently. One gene pair
$\left(\operatorname{traD} D_{T i}\right)$ has $K_{\mathrm{s}}=1.89$, a figure compatible with the overall median $K_{\mathrm{s}}$ of true orthologs. The two remaining genes (histidine kinase and spoT6 genes) have relatively high $K_{\mathrm{s}}$ values $\left(K_{\mathrm{s}}=2.90\right.$ and 5.57, respectively) and are responsible for the highest peak of the median $K_{\mathrm{s}}$ curve. These two genes probably experienced different evolutionary trajectories than the rest of the tra cluster, as already evidenced in their respective phylogenetic reconstructions (Fig. 3, cf. I and K). These sequences diverged seemingly earlier than the split between $R$. bellii and $R$. massiliae.

To further investigate the origin of the tra cluster genes, we analyzed the nucleotide composition of the $R$. massiliae and $R$. bellii genes using the Bayesian method developed by Nakamura et al. (2004). The R. massiliae tra cluster genes have collectively a lower horizontal transfer index (HTI), causing a small drop in the HTI median curve (Fig. 4B, red curve). This indicates that these genes exhibit a poorer fit to the average genome composition. Conversely, the horizontal transfer donor index (HTDI) median curve calculated using the $R$. bellii composition model exhibit a conspicuous peak centered on the tra region (Fig. 4B, blue curve). This indicates that those genes have a better fit with the average $R$. bellii gene nucleotide composition than does the rest of the genome. In contrast, the $R$. bellii tra cluster genes present neither sensible deviation from the average $R$. bellii nucleotide composition (HTI curve; Fig. 4C) nor a better fit to the $R$. massiliae model (HTDI curve; Fig. 4C). Altogether, these data strongly suggest that a recent $R$. massiliae ancestor has acquired the tra gene cluster through horizontal transfer from another Rickettsia species closely related to $R$. bellii. 


\section{The tra regions are free from RPE}

The $R$. massiliae genome contains 574 identified repeated elements (see Supplemental Material), most (98\%) of which belong to the repeated palindromic element (RPE) families (Ogata et al. 2001; Claverie and Ogata 2003). All Rickettsia species sequenced so far are littered with RPE sequences throughout their genomes. In $R$. massiliae, there is on average one repeat unit every $2.4 \mathrm{~kb}$, distributed largely homogeneously across the intergenic spacers of the genome and in some functional genes (RPEs constitute most red and green dots out of the main diagonal in Fig. 1). Intriguingly, the tra cluster region is located within by far the largest $(63.5 \mathrm{~kb})$ RPE-free segment of the genome (the second largest is $15.8 \mathrm{~kb}$ ). This segment appears as an empty vertical stripe in Figure 1. In $R$. bellii, the tra cluster is also part of the largest $(45.3 \mathrm{~kb}$ ) RPE-free region (average density is 1 repeat every $2.9 \mathrm{~kb}$ ). The $R$. africae and $R$. canadensis tra regions also contain no RPEs. The lack of RPEs is unlikely to result from strong selective pressures that forced the tra region to remain RPE-free, as most of the genes are not a priori involved in vital biological processes. Furthermore, the intergenic regions where most of the RPEs normally lie are not smaller in the tra region than in the rest of the genome. Thus the absence of RPEs suggests that the primary rickettsial tra gene cluster was most likely acquired after the RPE proliferation in Rickettsia.

\section{Discussion}

Although R. massiliae is one of the earliest diverging species of the SFG rickettsiae, its genome is very similar to the genomes of other SFG rickettsiae, in terms of sequence and gene order. Nevertheless, our analysis unveiled interesting new features, especially the origin of the tra cluster first observed in $R$. bellii (Ogata et al. 2006) and the existence of another rickettsial plasmid. The discovery of the tra cluster in $R$. bellii was followed by the identification of sex pili-like appendages, supporting the hypothesis that the tra genes are indeed involved in conjugal DNA transfer machinery. Those appendages are also present at the surface of $R$. massiliae cells (Supplemental Fig. S10).

As much as horizontal gene transfer (HGT) is a major contributor of genome evolution in free-living bacteria, the absence of (evidence of) HGT has long been considered a fundamental characteristic of genome evolution in obligate intracellular bacteria. The small genomes and isolated lifestyles of obligate intracellular bacteria are thought to reduce the opportunities for gene acquisition. It has also been hypothesized that genetic exchanges are minored in these species because their relatively constant intracellular environment does not select for the genetic diversity promoted by more challenging environments (Moreno 1998). These early views are now progressively changing with the accumulation of genomic data, which provide evidence for a better appraisal of the nature and extent of genetic exchange in intracellular bacteria (Bordenstein and Reznikoff 2005).

The Rickettsia genus has been shown to contain different families of mobile elements, such as plasmids (Ogata et al. 2005), transposases, and phage-related genes (Andersson et al. 1998; Ogata et al. 2001, 2005, 2006; McLeod et al. 2004). While a substantial number of HGT was inferred between the early ancestors of Rickettsiales and amoebal parasites (Ogata et al. 2006), our previous study did not identify clear evidence for more recent HGT events in the Rickettsia genomes (Blanc et al. 2007). However, this study was principally tailored for identifying HGT be- tween Rickettsia sp. and species outside the Rickettsia genus. Thus, HGT involving Rickettsia species both as donor and recipient were overlooked. In the present study, we demonstrate that $R$. massiliae received a 54.6-kb genetic element containing 44 genes from a species related to $R$. bellii. Furthermore, we also present evidence that other rickettsiae contain traces of genetic elements related to the $R$. massiliae tra cluster or rickettsiae plasmids. Our analysis suggests that these elements were acquired through distinct integration events and horizontal transfers between Rickettsia species (Fig. 3). Thus the $R$. massiliae tra cluster may be a member of a larger family of rickettsial conjugative elements that integrate specifically into the $t R N A$-Val gene owing to the integrase site specificity. We also note that the 12-kb R. prowazekiispecific DNA region (McLeod et al. 2004) contains genes that are otherwise only found in rickettsial plasmids. This region results probably from the recent (i.e., after the separation with $R$. typhi) integration of a rickettsial plasmid into the genome. Other studies have also presented evidence of evolutionary events related to HGT: the replacement of the ancestral $R$. felis and $R$. akari pat1 gene by a sequence closely related to the $R$. felis plasmid pat 2 gene (Blanc et al. 2005) and recombination of sca genes between different Rickettsia species (Jiggins 2006).

HGT in obligate intracellular bacteria is not limited to the Rickettsia genus, although evidence remains scarce in comparison to free-living bacteria. The Buchnera aphicolada genomes, estimated to have diverged 50-70 million years ago, exhibit identical genomic architectures and no indication of HGT (Tamas et al. 2002). This genomic stasis is thought to result from the loss of DNA repair and recombinase function (Moran and Wernegreen 2000; Dale et al. 2003). Nevertheless, evidence indicate that the Buchnera leuABCD plasmid might experience rare horizontal transmission (Van Ham et al. 2000) and genetic exchange with the Buchnera chromosome (Sabater-Munoz et al. 2004). Wolbachia is a genus of intracellular bacteria phylogenetically related to Rickettsia. The genome sequence from Wolbachia pipientis wMel is littered with repetitive and mobile DNA sequences, including numbers of prophage families ( $\mathrm{Wu}$ et al. 2004). One of these prophage, WO-B, can be horizontally transferred between different Wolbachia subgroups when they co-infect the same host (Masui et al. 2000; Bordenstein and Wernegreen 2004; Baldo et al. 2006). Recently, the O. tsutsugamushi genome revealed an astonishing number of mobile sequences, including $>400$ transposase genes and $>24$ tra gene clusters resulting from intragenomic duplications and/or multiple integrations (Cho et al. 2007).

Evidence suggests that the Rickettsiales ancestor lived in amoeba-like ancestral protozoa, which promoted genetic exchanges with other amoebal parasites and the adaptation to intracellular life in eukaryotes (Ogata et al. 2006). The horizontal transfer of the tra-region occurred between two lineages whose extant representatives live in ticks. As far as the life styles of $R$. massiliae and $R$. bellii can be extended to their recent ancestors, the exchange of the tra cluster probably occurred between bacteria that co-infected the same tick. Preliminary studies show that $R$. bellii can coexist with other rickettsiae in ticks in the wild (D. Raoult, unpubl.). Thus, the pattern of identifiable HGT in Rickettsia validates the "intracellular arena" hypothesis (Bordenstein and Wernegreen 2004), which posits that genetic material can move in and out of communities of obligate intracellular bacteria that co-infect the same intracellular host environment.

Conjugative elements are present in a variety of Grampositive and -negative bacteria and probably contribute as much as plasmids to the spread of antibiotic resistance genes in some

\section{Genome Research}

www.genome.org 
genera of disease-causing bacteria (Salyers et al. 1995). In the case of Rickettsia, the tra cluster includes members of the most prominent multigene families, such as the spoT (hydrolase domain), proline/betaine transporter, and toxin-antitoxin genes, which may have important functions with regard to the interaction with the eukaryotic host (Ogata et al. 2005). The spoT and proline/betaine transporter genes are often found as adjacent gene pairs in the Rickettsia genomes as well as in the consensus structure of the rickettsial tra region (Table 1). Toxin and antitoxin genes are also customarily organized in tandem. These gene pairs might have been hitchhiked between co-infecting Rickettsia species via ancient rickettsial genetic elements (for which few traces remain in the genomes today), thus promoting the adaptation to the host.

\section{Methods}

\section{Bacterial purification and DNA extraction}

Rickettsia massiliae (strain MTU5) was cultivated on L929 cells growing on EMEM (Biowhittaker, Cambrex Biosciences) supplemented with $2 \mathrm{mM}$ glutamine and $4 \%$ fetal calf serum (GIBCO, Invitrogen) in $150-\mathrm{cm}^{2}$ tissue culture flasks at $32^{\circ} \mathrm{C}$. The cultures were first harvested and treated by trypsin to a final concentration of $1 \%$ followed by a digestion with DNase I. The final purification was performed by two Renografin density gradient centrifugations. The pellet of purified $R$. massiliae was again digested three times by DNase I. Prokaryotic DNA was extracted according to the classical lytic treatment using SDS and proteinase $\mathrm{K}$ followed by phenol-chloroform-isoamyl alcohol extraction; DNA was resuspended in TE buffer and visualized on agarose gel stained by ethidium bromide.

\section{Shotgun sequencing of $R$. massiliae genome}

Genomic DNA was fragmented by mechanical shearing using a Hydroshear device (GeneMachine). A preliminary library (3-kb inserts cloned in pcDNA 2.1 with BstXI adaptors) was constructed and validated. The percentage of eukaryotic DNA contamination was calculated from the sequence on 96 clones and was $<7 \%$. We generated another library with $10-\mathrm{kb}$ inserts cloned in the pCNS vector (C. Robert, unpubl.). Plasmid clones were sequenced at both ends of the insert with flanking vector sequences as primers. Dye-terminator reactions were analyzed on a capillary ABI3730 (Applied Biosystems). The whole-genome sequence assembly was performed by means of the PHRED and PHRAP software packages. Cloning gaps and sensitive regions were resolved on subclones templates or confirmed by sequencing PCR products; 579 primers were involved in the finishing. The shotgun sequence assembly revealed a plasmid of $\sim 16 \mathrm{~kb}$. Its presence was confirmed by Southern blot. The sequences have been deposited in the GenBank database (accession nos. CP000683 and CP000684).

\section{Sequence analysis}

We predicted protein coding genes (ORFs) using SelfID as previously described (Ogata et al. 2005). tRNA genes were identified using tRNAscan-SE (Malek et al. 2004). Database searches were performed using BLAST programs against SWISS-PROT/TrEMBL (Apweiler et al. 2004), NCBI/CDD database (Marchler-Bauer et al. 2005), and SMART (Ponting et al. 1999). Representation of the BLAST results, as in Figure 2, was performed using an in-house Perl script available upon request from the author. Repeated DNA sequences were identified using RepeatFinder (Volfovsky et al. 2001). To identify Rickettsia palindromic elements, we used hid- den Markov models (Eddy 1996) based on previously identified RPE sequences.

We used MUSCLE (Edgar 2004) to perform multiple sequence alignments. Maximum-likelihood phylogenetic analyses were conducted using PHYML (Guindon and Gascuel 2003) after manual edition of the alignments to remove poorly aligned regions and sites containing gaps. We used the ProtTest program to select the substitution models that best describe the data using the AIC criterion (Abascal et al. 2005). We applied the Shimodaira-Hasegawa (SH) test (Shimodaira and Hasegawa 1999) as implemented in the TREE-PUZZLE program (Schmidt et al. 2002) to verify whether the maximum-likelihood tree topologies were significantly better at explaining the data than the expected species-tree topology. The true phylogenetic position of the $R$. felis plasmid sequences is unknown and can change from gene to gene because of the composite nature of plasmids. Thus, we omitted the $R$. felis sequence in the $\mathrm{SH}$ test. $K_{\mathrm{s}}$ values were estimated from pairs of codon-aligned nucleotide sequences using the CODEML program (Yang 1997). HTI and HTDI values for genes were determined using a computer program developed by Nakamura et al. (2004): Markov chain models for nucleotide composition were constructed for the $R$. massiliae and $R$. bellii genomes and then used to calculate the average posterior probability (i.e., index) for each gene sequence to be compatible with the rest of the genome. Low horizontal index values indicate poor fit with the tested genome. We used a Markov order of 5 with a 96-bp window sliding with a step size of $12 \mathrm{bp}$.

\section{Acknowledgments}

We thank Prof. Y. Nakamura for providing us with the computer program that computes the horizontal gene transfer index.

\section{References}

Abascal, F., Zardoya, R., and Posada, D. 2005. ProtTest: Selection of best-fit models of protein evolution. Bioinformatics 21: 2104-2105. Andersson, S.G., Zomorodipour, A., Andersson, J.O., Sicheritz-Ponten, T., Alsmark, U.C., Podowski, R.M., Naslund, A.K., Eriksson, A.S., Winkler, H.H., and Kurland, C.G. 1998. The genome sequence of Rickettsia prowazekii and the origin of mitochondria. Nature 396: $133-140$.

Apweiler, R., Bairoch, A., Wu, C.H., Barker, W.C., Boeckmann, B., Ferro, S., Gasteiger, E., Huang, H., Lopez, R., Magrane, M., et al. 2004. UniProt: The Universal Protein knowledgebase. Nucleic Acids Res. 32: D115-D119. doi: 10.1093/nar/gkh131.

Baldo, L., Bordenstein, S., Wernegreen, J.J., and Werren, J.H. 2006. Widespread recombination throughout Wolbachia genomes. Mol. Biol. Evol. 23: 437-449.

Beati, L. and Raoult, D. 1993. Rickettsia massiliae sp. nov., a new spotted fever group Rickettsia. Int. J. Syst. Evol. Microbiol. 43: 839-840.

Blanc, G., Renesto, P., and Raoult, D. 2005. Phylogenic analysis of rickettsial Patatin-like protein with conserved phospholipase $\mathrm{A}_{2}$ active sites. Ann. N.Y. Acad. Sci. 1063: 83-86.

Blanc, G., Ogata, H., Robert, C., Audic, S., Suhre, P.K., Vestris, G., Claverie, J.-M., and Raoult, D. 2007. Reductive genome evolution from the mother of Rickettsia. PLoS Genet. 3: e14. doi: 10.1371/journal.pgen.0030014.

Bordenstein, S.R. and Reznikoff, W.S. 2005. Mobile DNA in obligate intracellular bacteria. Nat. Rev. Microbiol. 3: 688-699.

Bordenstein, S.R. and Wernegreen, J.J. 2004. Bacteriophage flux in endosymbionts (Wolbachia): Infection frequency, lateral transfer, and recombination rates. Mol. Biol. Evol. 21: 1981-1991.

Burrus, V., Pavlovic, G., Decaris, B., and Guedon, G. 2002. Conjugative transposons: The tip of the iceberg. Mol. Microbiol. 46: 601-610.

Cardenosa, N., Segura, F., and Raoult, D. 2003. Serosurvey among Mediterranean spotted fever patients of a new spotted fever group rickettsial strain (Bar29). Eur. J. Epidemiol. 18: 351-356.

Cho, N.-H., Kim, H.-R., Lee, J.-H., Kim, S.-Y., Kim, J., Cha, S., Kim, S.-Y., Darby, A.C., Fuxelius, H.-H., Yin, J., et al. 2007. The Orientia tsutsugamushi genome reveals massive proliferation of conjugative 
type IV secretion system and host-cell interaction genes. Proc. Natl. Acad. Sci. 104: 7981-7986.

Claverie, J.M. and Ogata, H. 2003. The insertion of palindromic repeats in the evolution of proteins. Trends Biochem. Sci. 28: 75-80.

Dale, C., Wang, B., Moran, N., and Ochman, H. 2003. Loss of DNA recombinational repair enzymes in the initial stages of genome degeneration. Mol. Biol. Evol. 20: 1188-1194.

Drancourt, M. and Raoult, D. 1999. Characterization of mutations in the $r p o B$ gene in naturally rifampin-resistant Rickettsia species. Antimicrob. Agents Chemother. 43: 2400-2403.

Eddy, S.R. 1996. Hidden Markov models. Curr. Opin. Struct. Biol. 6: $361-365$.

Edgar, R.C. 2004. MUSCLE: Multiple sequence alignment with high accuracy and high throughput. Nucleic Acids Res. 32: 1792-1797. doi: 10.1093/nar/gkh340.

Eremeeva, M.E., Madan, A., Shaw, C.D., Tang, K., and Dasch, G.A. 2005 New perspectives on rickettsial evolution from new genome sequences of Rickettsia, particularly $R$. canadensis, and Orientia tsutsugamushi. Ann. N.Y. Acad. Sci. 1063: 47-63.

Eremeeva, M.E., Bosserman, E.A., Demma, L.J., Zambrano, M.L., Blau, D.M., and Dasch, G.A. 2006. Isolation and identification of Rickettsia massiliae from Rhipicephalus sanguineus ticks collected in Arizona. Appl. Environ. Microbiol. 72: 5569-5577.

Fernandez-Soto, P., Perez-Sanchez, R., Diaz Martin, V., Encinas-Grandes, A., and Alamo Sanz, R. 2006. Rickettsia massiliae in ticks removed from humans in Castilla y Leon, Spain. Eur. J. Clin. Microbiol. Infect. Dis. 25: $811-813$.

Fournier, P.E., Roux, V., and Raoult, D. 1998. Phylogenetic analysis of spotted fever group rickettsiae by study of the outer surface protein rOmpA. Int. J. Syst. Evol. Microbiol. 48: 839-849.

Guindon, S. and Gascuel, O. 2003. A simple, fast, and accurate algorithm to estimate large phylogenies by maximum likelihood. Syst. Biol. 52: 696-704.

Jiggins, F.M. 2006. Adaptive evolution and recombination of Rickettsia antigens. J. Mol. Evol. 62: 99-110.

Malek, J.A., Wierzbowski, J.M., Tao, W., Bosak, S.A., Saranga, D.J., Doucette-Stamm, L., Smith, D.R., McEwan, P.J., and McKernan, K.J. 2004. Protein interaction mapping on a functional shotgun sequence of Rickettsia sibirica. Nucleic Acids Res. 32: 1059-1064. doi: $10.1093 /$ nar/gkh254.

Marchler-Bauer, A., Anderson, J.B., Cherukuri, P.F., DeWeese-Scott, C., Geer, L.Y., Gwadz, M., He, S., Hurwitz, D.I., Jackson, J.D., Ke, Z., et al. 2005. CDD: A conserved domain database for protein classification. Nucleic Acids Res. 33: D192-D196. doi: $10.1093 / \mathrm{nar} / \mathrm{gki069}$

Masui, S., Kamoda, S., Sasaki, T., and Ishikawa, H. 2000. Distribution and evolution of bacteriophage WO in Wolbachia, the endosymbiont causing sexual alterations in arthropods. J. Mol. Evol. 51: $491-497$.

Matsumoto, K., Ogawa, M., Brouqui, P., Raoult, D., and Parola, P. 2005. Transmission of Rickettsia massiliae in the tick, Rhipicephalus turanicus. Med. Vet. Entomol. 19: 263-270.

McLeod, M.P., Oin, X., Karpathy, S.E., Gioia, J., Highlander, S.K., Fox, G.E., McNeill, T.Z., Jiang, H., Muzny, D., Jacob, L.S., et al. 2004. Complete genome sequence of Rickettsia typhi and comparison with sequences of other rickettsiae. J. Bacteriol. 186: 5842-5855.

Moran, N.A. and Wernegreen, J.J. 2000. Lifestyle evolution in symbiotic bacteria: insights from genomics. Trends Ecol. Evol. 15: 321-326.

Moreno, E. 1998. Genome evolution within the alpha Proteobacteria: Why do some bacteria not possess plasmids and others exhibit more than one different chromosome? FEMS Microbiol. Rev. 22: 255-275.

Nakamura, Y., Itoh, T., Matsuda, H., and Gojobori, T. 2004. Biased biological functions of horizontally transferred genes in prokaryotic genomes. Nat. Genet. 36: 760-766.

Ogata, H., Audic, S., Renesto-Audiffren, P., Fournier, P.-E., Barbe, V., Samson, D., Roux, V., Cossart, P., Weissenbach, J., Claverie, J.-M., et al. 2001. Mechanisms of evolution in Rickettsia conorii and $R$. prowazekii. Science 293: 2093-2098.
Ogata, H., Renesto, P., Audic, S., Robert, C., Blanc, G., Fournier, P.E. Parinello, H., Claverie, J.M., and Raoult, D. 2005. The genome sequence of Rickettsia felis identifies the first putative conjugative plasmid in an obligate intracellular parasite. PLoS Biol. 3: e248. doi: 10.1371/journal.pbio.0030248

Ogata, H., La Scola, B., Audic, S., Renesto, P., Blanc, G., Robert, C., Fournier, P.E., Claverie, J.M., and Raoult, D. 2006. Genome sequence of Rickettsia bellii illuminates the role of amoebae in gene exchanges between intracellular pathogens. PLoS Genet. 2: e76. doi: 10.1371/journal.pgen.0020076.

Parola, P., Paddock, C.D., and Raoult, D. 2005. Tick-borne rickettsioses around the world: Emerging diseases challenging old concepts. Clin. Microbiol. Rev. 18: 719-756.

Ponting, C.P., Schultz, J., Milpetz, F., and Bork, P. 1999. SMART: Identification and annotation of domains from signalling and extracellular protein sequences. Nucleic Acids Res. 27: 229-232.

Raoult, D. and Roux, V. 1997. Rickettsioses as paradigms of new or emerging infectious diseases. Clin. Microbiol. Rev. 10: 694-719.

Rolain, J.M., Maurin, M., Vestris, G., and Raoult, D. 1998. In vitro susceptibilities of 27 Rickettsiae to 13 antimicrobials. Antimicrob. Agents Chemother. 42: 1537-1541.

Roux, V., Rydkina, E., Eremeeva, M., and Raoult, D. 1997. Citrate synthase gene comparison, a new tool for phylogenetic analysis, and its application for the rickettsiae. Int. J. Syst. Bacteriol. 47: 252-261.

Sabater-Munoz, B., van Ham, R.C.H.J., Moya, A., Silva, F.J., and Latorre, A. 2004. Evolution of the leucine gene cluster in Buchnera aphidicola: Insights from chromosomal versions of the cluster. J. Bacteriol. 186: $2646-2654$.

Salyers, A.A., Shoemaker, N.B., Stevens, A.M., and Li, L.Y. 1995. Conjugative transposons: An unusual and diverse set of integrated gene transfer elements. Microbiol. Rev. 59: 579-590.

Schmidt, H.A., Strimmer, K., Vingron, M., and von Haeseler, A. 2002. TREE-PUZZLE: Maximum likelihood phylogenetic analysis using quartets and parallel computing. Bioinformatics 18: 502-504.

Shimodaira, H. and Hasegawa, M. 1999. Multiple comparisons of log-likelihoods with applications to phylogenetic inference. Mol. Biol. Evol. 16: 1114-1116.

Stothard, D.R., Clark, J.B., and Fuerst, P.A. 1994. Ancestral divergence of Rickettsia bellii from the spotted fever and typhus groups of Rickettsia and antiquity of the genus Rickettsia. Int. J. Syst. Bacteriol. 44: 798-804.

Tamas, I., Klasson, L., Canback, B., Naslund, A.K., Eriksson, A.S., Wernegreen, J.J., Sandstrom, J.P., Moran, N.A., and Andersson, S.G. 2002. 50 Million years of genomic stasis in endosymbiotic bacteria. Science 296: 2376-2379.

Van Ham, R.C., Gonzalez-Candelas, F., Silva, F.J., Sabater, B., Moya, A., and Latorre, A. 2000. Postsymbiotic plasmid acquisition and evolution of the repA1-replicon in Buchnera aphidicola. Proc. Natl. Acad. Sci. 97: 10855-10860.

Vitale, G., Mansueto, S., Rolain, J.M., and Raoult, D. 2005. Rickettsia massiliae human isolation. Emerg. Infect. Dis. 12: 174-175.

Volfovsky, N., Haas, B.J., and Salzberg, S.L. 2001. A clustering method for repeat analysis in DNA sequences. Genome Biol. 2: RESEARCH0027. doi: 10.1186/gb-2001-2-8-research0027.

Williams, K.P. 2002. Integration sites for genetic elements in prokaryotic tRNA and tmRNA genes: Sublocation preference of integrase subfamilies. Nucleic Acids Res. 30: 866-875.

Wu, M., Sun, L.V., Vamathevan, J., Riegler, M., Deboy, R., Brownlie, J.C., McGraw, E.A., Martin, W., Esser, C., Ahmadinejad, N., et al. 2004. Phylogenomics of the reproductive parasite Wolbachia pipientis wMel: A streamlined genome overrun by mobile genetic elements. PLoS Biol. 2: e69. doi: 10.1371/journal.pbio.0020069.

Yang, Z. 1997. PAML: A program package for phylogenetic analysis by maximum likelihood. Comput. Appl. Biosci. 13: 555-556.

Received May 25, 2007; accepted in revised form August 29, 2007. 


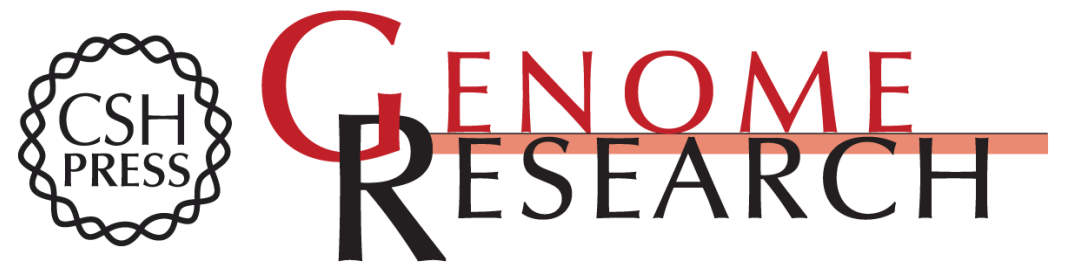

\section{Lateral gene transfer between obligate intracellular bacteria: Evidence from the Rickettsia massiliae genome}

Guillaume Blanc, Hiroyuki Ogata, Catherine Robert, et al.

Genome Res. 2007 17: 1657-1664 originally published online October 4, 2007

Access the most recent version at doi:10.1101/gr.6742107

Supplemental Material

References

License

Email Alerting Service
http://genome.cshlp.org/content/suppl/2007/10/05/gr.6742107.DC1

This article cites 51 articles, 12 of which can be accessed free at: http://genome.cshlp.org/content/17/11/1657.full.html\#ref-list-1

Receive free email alerts when new articles cite this article - sign up in the box at the top right corner of the article or click here.

\section{Affordable, Accurate Sequencing.}

